\title{
Novel extracorporeal membrane oxygenation support strategy as a treatment for acute lung injury following ventricular assist device implantation
}

\author{
Vander Pluym CJ*1, Daly KP', Thiagarajan $\mathrm{R}^{2}$ and Fynn-Thompson $\mathrm{F}^{2}$ \\ ${ }^{1}$ Departments of Cardiology and Cardiac Surgery, Boston Children's Hospital, Boston, MA, 02115, USA \\ ${ }^{2}$ Department of Pediatrics and Department of Cardiac Surgery, Harvard Medical School, 300 Longwood Avenue, Boston, MA, 02115, USA
}

\section{Case report}

Acute lung injury (ALI) presenting as fulminant pulmonary edema has been described as a complication following conversion from extracorporeal membrane oxygenation (ECMO) to ventricular assist device (VAD) support. ${ }^{1}$ The exact mechanism for this phenomenon is unclear and it portends a poor prognosis, with reported mortality of 87\% [1]. We describe a case of ALI following ECMO conversion to biventricular VAD support in a child that was successfully managed by a novel ECMO reconfiguration as a bridge-to-bridge strategy.

A 2 year old $9.2 \mathrm{~kg}$ (BSA $0.46 \mathrm{~m}^{2}$ ) female awaiting heart transplant for restrictive cardiomyopathy was emergently placed on veno-arterial (VA) ECMO within 45 minutes of witnessed in-hospital cardiac arrest. Standard cannulation with $10 \mathrm{Fr}$ common carotid arterial canulla and a 14Fr internal jugular venous cannula achieved cardiac index of 3 $\mathrm{L} / \mathrm{min} / \mathrm{m}^{2}$. Post-operative echocardiogram demonstrated severely dilated and dysfunctional left ventricle with no patent foramen ovale. Severe pulmonary edema on chest roentogram (CXR) with pulmonary hemorrhage prompted emergent cardiac catheterization for decompression of left atrium (LA). Catheterization demonstrated no antegrade pulmonary blood flow with severe elevation and equalization of pulmonary arterial (PA) and LA pressures at $44 \mathrm{mmHg}$. Following atrial septal stenting, the LAP decreased to $20 \mathrm{mmHg}$ with angiographic evidence of antegrade pulmonary flow.

Over the course of 10 days, pulmonary edema improved drastically on CXR. Normalization of her end organ function prompted conversion to biventricular assist device as a bridge to transplantation. Through a median sternotomy approach, $6 \mathrm{~mm}$ inflow Berlin Heart ${ }^{\circledR}$ atrial cannula were placed in the RA and LA, with $6 \mathrm{~mm}$ outflow Berlin Heart ${ }^{\circledR}$ arterial cannula placed in the main pulmonary artery (MAP) and ascending aorta (AsAo) to provide right and left VAD support, respectively. The cannulas were externalized through the abdomen and connected to 2 continuous flow (cf) centrifugal Rotaflow ${ }^{\circledR}$ pumps. Within 48 hours, she developed progressive pulmonary edema and increase in central venous pressures from 10 to $20 \mathrm{mmHg}$ that were refractory to optimization of ventilation strategies, increase in VAD operating speed, diuresis and paralysis. Due to hypoxemia, an oxygenator was interposed after the LVAD. However progressive decline in LVAD flow necessitated RV support resulting in conversion of the mechanical circulatory support (MCS) strategy.

Our novel ECMO cannulation and management strategy included joining the RA and LA Berlin Heart ${ }^{\circledR}$ venous cannulas together and connect them to a single Rotaflow ${ }^{\circledR}$ pump followed by a Quadrox ${ }^{\mathbb{R}}$ oxygenator, with dual outflow to the PA and AsAo Berlin Heart ${ }^{\mathbb{B}}$ arterial cannula. (Figure 1) We placed a clamp over the external segment of the PA outflow cannula to restrict the amount of blood going to the pulmonary circulation $(\mathrm{Qp})$. Flow probes were placed over the PA cannula distal to the clamp, over the LA cannula and over the AsAo cannula, allowing calculation of the Qp:Qs. Starting with a measured Qp:Qs of 0.2:1, (total flows of $3 \mathrm{~L} / \mathrm{min} / \mathrm{m}^{2}$ ), we subsequently increased the Qp by increments of 0.2 over the course of a week by decreasing the clamp tension, until Qp:Qs was 1:1. By day 11 of support, she had complete resolution of pulmonary edema with significant improvement in lung tidal volumes on standard ventilation settings. Sampling of pulmonary venous blood through her LA cannula demonstrated capacity for gas exchange with weaning of oxygenator

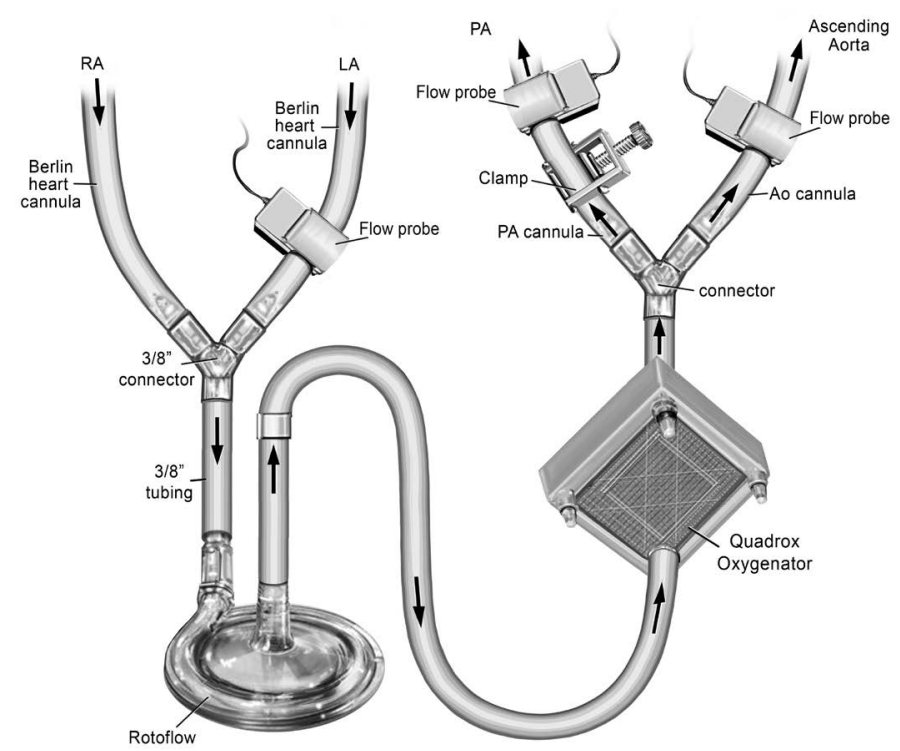

Figure 1. Novel Extracorporeal Membrane Oxygenation Configuration to provide gradual increase in pulmonary blood flow for treatment of acute lung injury.

Correspondence to: VanderPluym CJ, Departments of Cardiology \& Cardiac Surgery, Boston Children's Hospital, Boston, MA, 02115, USA

Received: November 28, 2016; Accepted: December 14, 2016; Published: December 19, 2016 
sweep gases. She was then converted back to BiVAD Rotaflow ${ }^{\circledR}$ devices with maintenance of the oxygenator distal to the LVAD. Sweep gases through the oxygenator were weaned over the next 24 hours, with planned removal of the oxygenator from the LVAD circuit. With recovery of end organ function, she was listed for heart transplant (HT) and underwent HT after an additional 5 days of BiVAD support (29 days total support).

The development of post-implant ALI following ECMO support is a devastating complication with limited treatment strategies. One possible hypothesis is that hypoperfusion of the pulmonary vascular bed leads to endothelial damage and that reestablishment of antegrade pulmonary blood flow leads to reperfusion injury, edema, and ALI As such, the ability to re-introduce pulmonary blood flow in a graded fashion with along with the assistance of an oxygenator may help mitigate ALI in patients thought to be at high risk (i.e. pulmonary edema on ECMO support, incomplete decompression of LA and LV during support, multiple transfusions) [1]. Further evaluation of this MCS strategy as a bridge to bridge is required.

\section{References}

1. Boulate D, Luyt CE, Pozzi M, Niculescu M, Combes A, et al. (2013) Acute lung injury after mechanical circulatory support implantation in patients on extracorporeal life support: An unrecognized problem. European Journal of Cardio-Thoracic Surgery: Official Journal of the European Association for Cardio-thoracic Surgery 44: 544-549.

Copyright: (C2016 Vander Pluym CJ. This is an open-access article distributed under the terms of the Creative Commons Attribution License, which permits unrestricted use, distribution, and reproduction in any medium, provided the original author and source are credited. 